\title{
Observational signs of planet infall and Roche lobe overflow outward migration
}

\author{
Stuart F. Taylor ${ }^{1,2,3}$ and Ing-Guey Jiang ${ }^{1}$ \\ ${ }^{1}$ Institute of Astronomy and Department of Physics, National Tsing Hua University, \\ 101 Section 2 Kuang Fu Road, Hsinchu, Taiwan 30013 \\ email: astrostuart@gmail.com, jiang@phys.nthu.edu.tw \\ ${ }^{2}$ Global Telescope Science Group, Los Angeles, California, USA \\ ${ }^{3}$ Eureka Scientific, Inc., Oakland, California, USA
}

\begin{abstract}
Outward migration of planets due to Roche lobe overflow may play an important role in producing the presently observed distribution of planet parameters. We suggest that many of the currently known short period planets may have already migrated into the Roche distance from the star, and then deposited at their current semi-major axes by being migrated outwards due to angular momentum transfer from an episode of Roche lobe overflow (RLO). This RLO outward migration (RLOOM) could be sustained in the region where planetary radius increases with decreasing mass. We are modeling how RLOOM may leave what kind of planet parameter statistics. This modeling seeks to predict what observable signs of RLOOM there may be. Overflow of planetary mass may leave behind characteristic hot dust and gas as well as produce luminous signatures.
\end{abstract}

Keywords. planetary systems, planetary systems: formation, stars: rotation, stars: statistics, stars: evolution, stars: variables: other

\section{Introduction}

The pile-up of planet orbits near 3 days (Fig. 1) has been a puzzle from the time there have been enough planet statistics to see patterns in orbital statistics (Santos \& Mayor 2003). Also, the radii of many but not all of these planets has been larger than expected (Gu 2010). Finally, some of these planets are reported have nonzero eccentricities despite strong eccentricity damping, though better measurements are needed. It is accepted that these close in planets must have formed further out and migrated inwards to their current position (Pont 2009). Efforts to explain these three mysteries have been based on trying to explain how these patterns could occur as these close in planets migrated inwards. Taylor (2010) and Fabrycky (2010)) suggest that the pile-up may be composed by planets that had migrated all the way to Roche lobe overflow (RLO), from where they had sufficient RLO to migrate outward (RLOOM), and that the end of RLOOM may have left planets in the pile-up. Though the role of outward migration following Roche lobe overflow (RLOOM) during the phase when the disk is still present has been studied (Chang \& Gu 2010; Gu, Bodenheimer, \& Lin 2004; Gu, Lin, \& Bodenheimer 2003; Trilling et al. 1998), there has been less study of RLOOM during the main sequence phase.

\section{Radius-mass relation maintaining outward migration}

The mass-radius relation for giant planets turns around due to electron degeneracy such that above a certain temperature-dependent mass, the planet radius begins to decrease with increasing mass (Zapolsky \& Salpeter 1969). This creates the potential instability 


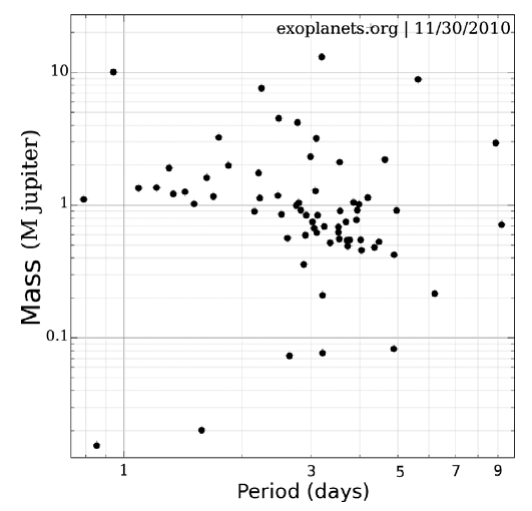

Figure 1. Mass versus period of the transiting planets in the region of the pile-up.

that when RLO removes mass the planet will expand its radius. RLO does not lead to RLOOM for "normal" planets that get smaller upon mass removal, because outward migration quenches the RLO as a planet's Roche radius increases with distance. Population modeling is in progress to see whether this can account for the observed pile-up (Taylor et al. in preparation). It must be determined whether the increase in radius due to mass loss is enough to fill the increasing size of the Roche lobe as the planet migrates outward.

If a tidal heating mechanism is necessary for RLOOM to operate sufficiently well to create the pile-up below one Jupiter mass, this may also explain how such planets might be left with a range of inflated radii. Because at the distance of RLO eccentricity is quickly damped out, it has been suggested that perhaps the process of RLOOM allows a means of input of eccentricity (Taylor 2010). Though an increase of RLOOM at periastron would translate into less rather than more eccentricity, work is exploring the range of possible patterns of delayed radius enlargement or other asymetric angular momentum transfer.

The most identifiable signal from past RLO may be the presence of dust and gas. How much depends on how much material is driven away from the star. The potential for a luminous signature from RLO or planet-star collisions has been emphasized (Taylor 2010), but it is also important to model the quantity of infalling dust and gas that may be driven outward to determine whether planetary material may leave an identifiable signal.

We acknowledge the support of National Tsing Hua University and the National Science Council of Taiwan. We made extensive use of the Exoplanet Data Explorer.

\section{References}

Chang, S.-H., Gu, P.-G., \& Bodenheimer, P. H. 2010, ApJ, 708, 1702

Fabrycky, D. 2010, This volume

Pont, F. 2010, MNRAS, 396, 1789

Gu, P.-G. 2010, Nature, 465, 300

Gu, P.-G., Lin, D. N. C., \& Bodenheimer, P. H. 2003, ApJ, 588, 509

Gu, P-.G., Bodenheimer, P. H., \& Lin, D. N. C. 2004, ApJ, 608, 1076

Santos, N. C. \& Mayor, M. 2003, in: M. Monteiro (ed.), The Unsolved Universe: Challenges for the Future, JENAM 2002 (Dordrecht: Kluwer Academic Publishers), p. 15

Taylor, S. F. 2010, submitted to Icarus, arXiv:1009.4221

Trilling, D. E., Benz, W., Guillot, T., Lunine, J. I., Hubbard, W. B., \& Burrows, A. 1998, ApJ, 500,428

Zapolsky, H. S. \& Salpeter, E. E. 1969, ApJ, 158, 809 